\title{
Wind energy research development of advanced design tools
}

\author{
- P.J. Eecen, H.A. Bijleveld and B. Sanderse - DOI: 10.1051/epn/2012203 \\ n Energy research Centre of the Netherlands ECN, Petten, the Netherlands
}

\section{Detailed aerodynamic computations are used to improve rotor blade design and to simulate wind turbine interactions in wind farms.}

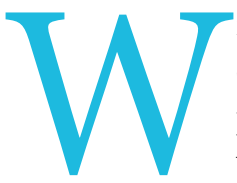

ind energy capacity has grown at a rate of approximately $30 \%$ per year over the past 20 years, as is indicated in Figure 1, yielding a cumulative installed capacity of $197 \mathrm{GW}$ world wide in 2010 [1]. This is despite the economic crisis, in which the financing of wind farms has become increasingly difficult. Europe, at the end of 2010 , had a total amount of $86 \mathrm{GW}$ wind energy capacity installed, producing 181 TWh of electricity on average and covering $5.3 \%$ of the European electricity demand (4.8\% in 2009). Wind energy nowadays plays an important role in the extension and replacement policy of power plants. Interestingly, in 2010 more wind energy capacity was installed in Asia than in the traditionally strong markets of
Europe and North America. China and India installed 19 GW and 2.1 GW respectively, whereas Europe and North America installed 9.9 GW and 5.8 GW, respectively. In Europe, the growth of onshore wind energy capacity was $13 \%(8.4 \mathrm{GW})$ while the growth in offshore wind energy capacity was 51\% (0.9 GW) compared with 2009.

At the end of 2010, the EU Renewable Energy Directive has been put into action, formalizing the set target for the EU of $20 \%$ renewables in the energy mix of 2020. Member states have submitted National Renewable Energy Action Plans to reach the 2020 target. Wind energy takes an important share; the Action Plans are based on 14\% wind energy contribution. The European Wind Energy Association (EWEA) [2] presented scenarios exceeding 
these numbers. In the context of reduction of $\mathrm{CO}_{2}$ in the atmosphere, wind energy is one of the preferred options. Depending on wind resources, it takes a wind turbine three to six months to recover the energy needed for building, operating and decommissioning the wind turbine over its 20 to 25 year operational lifetime.

Currently, the cost of electricity production by onshore wind turbines approaches the cost of fossil fuel electricity production ( 6 - $8 € \mathrm{ct} / \mathrm{kWh}$ ). Offshore, the cost of wind electricity strongly depends on the complexity of the wind farm, including distance to the coast, water depth and soil conditions. The cost of offshore wind energy electricity is currently in the order of $12-18 € \mathrm{ct} / \mathrm{kWh}$. However, one expects this relatively young offshore wind industry to experience a steep learning curve leading to significant cost reductions. European governments balance their funding between implementation and innovation. North Sea and Baltic Sea countries have a focus on further development of offshore wind energy. Other coastal countries like Greece, Spain, Italy and Norway investigate floating wind turbines because of their large depth coastal waters.

Wind turbines have become more cost effective over the years, whilst the size of turbines has grown. From dimensional analysis, up-scaling wind turbines will not automatically lead to a reduction of the cost of wind power. Power generated by a wind turbine scales as the square of the rotor diameter, while the mass scales with its cube. Up scaling would thus lead to increased cost of energy. The main reason that the cost of wind energy has come down with increased size wind turbines is continuous innovation. Recently, a large European project 'UPWIND' [3] has investigated the challenges and limitations for up scaling wind turbines to 20MW size. For offshore application of wind power new technologies and knowhow are needed beyond the existing knowledge base. Not only new technologies are required, also up scaling wind turbine dimensions, wind farm capacities and innovative electrical infrastructure is a requirement to make offshore wind energy a success. The push for offshore wind energy is the driver of current European wind energy research and is expected to also lead to more cost effective onshore installations.

In order to maintain Europe's global leadership in wind energy, more research is needed and the European research institutes have organized themselves in the European Energy Research Alliance (EERA) [4]. Its key objective is to accelerate the development of new energy technologies by drafting and implementing Joint Research Programmes in support of the Strategic Energy Technology (SET) plan [5] based on pooling and integrating activities and resources. The up scaling and increased complexity of wind turbines require more accurate design calculations, for example to reduce safety factors. The Energy research Centre of the Netherlands (ECN) is known for its development of design tools in wind energy. The aerodynamics research focuses firstly on the modeling of the wind turbine rotor

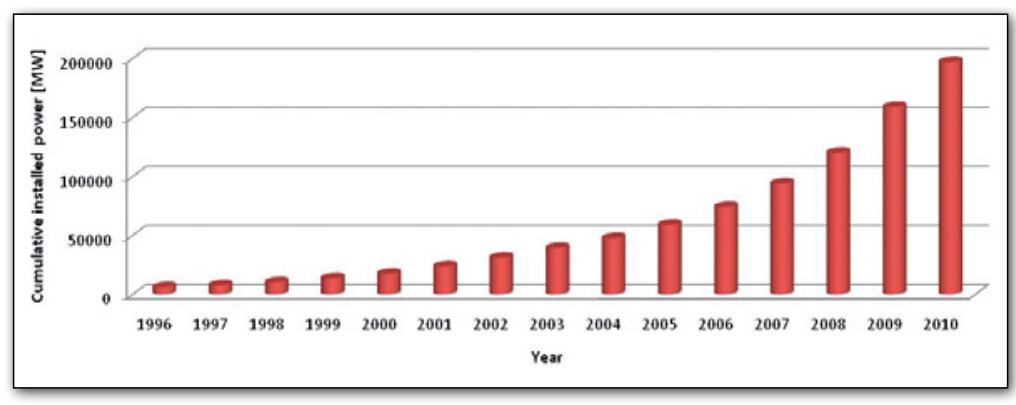

and secondly on the flow in and around wind farms. The generated knowledge is brought in the wind energy market offering design support and licensing of innovations. Two recent developments are described here.

\section{Rotor aerodynamics}

ECN traditionally holds a strong position in the field of rotor aerodynamics. Its research aims to incorporate the developed knowledge in aerodynamic design tools enabling increased accuracies in designing future large wind turbine blades. An important development at ECN is the AeroModule, a collection of advanced aerodynamic design tools coupling aerodynamic modeling with structural dynamics tools. An advanced Blade Element Momentum (BEM) model can just as easily be applied as the ECN free-vortex wake code called 'AWSM' [6]. With this tool the accuracy of integrated wind turbine designs can be evaluated, see Figure 2.

For the design of wind turbine blades an accurate prediction of the aerodynamic loading on the blade surface is necessary. With increased blade sizes the flexibility of the blades increases and unsteady aerodynamic effects become increasingly important. RotorFlow is a simulation code under development at ECN that can determine the aerodynamic forces on wind turbine blades for unsteady flow. The method will yield accurate results while keeping the computational time acceptable. $\triangle$ FIG 1: Global cumulative installed wind energy capacity [1]
V FIG 2:

The effect of the wind profile on the wake development the free-vortex wake code AWSM. as calculated with

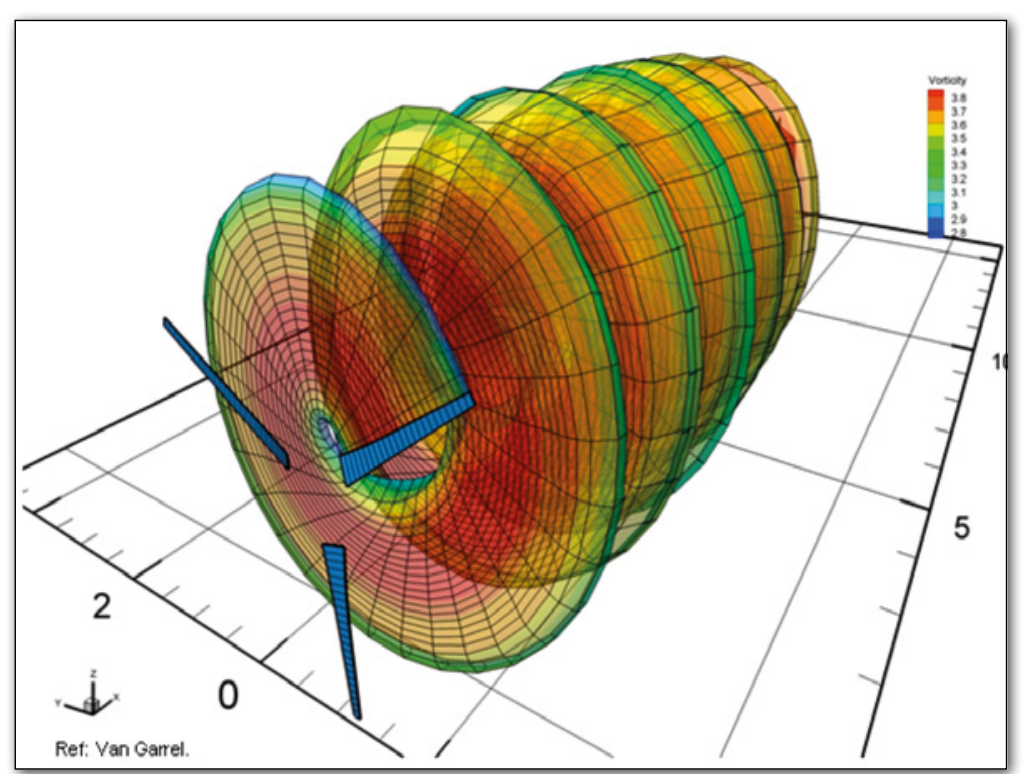


$\triangle$ FIG 3: Flow field decomposition into an inviscid and viscous region.
The flow field around the blade is split into an internal viscous region close to the surface and an external inviscid region, see Figure 3. The inviscid flow is solved using a panel method. The viscous flow is modeled as a boundary layer flow and solved with a DiscontinuousGalerkin scheme for non-conservative systems of partial differential equations. To ensure a proper match between the two flow regions, a quasi-simultaneous interaction scheme is applied. This scheme, developed by Veldman [7], is extremely suitable for this application as it is robust - even in separated flows - and easy in terms of programming. The boundary layer equations are solved together with the interaction law equations from the interaction method. The interaction law equations are a very crude approximation of the inviscid flow taking only the local influence in account.

In RotorFlow the inviscid flow and the viscous flow with the interaction scheme are solved iteratively where the values of variables describing the flow are exchanged, see Figure 4. The separate components of RotorFlow have been tested independently [8] and show promising results for the final implementation in RotorFlow.

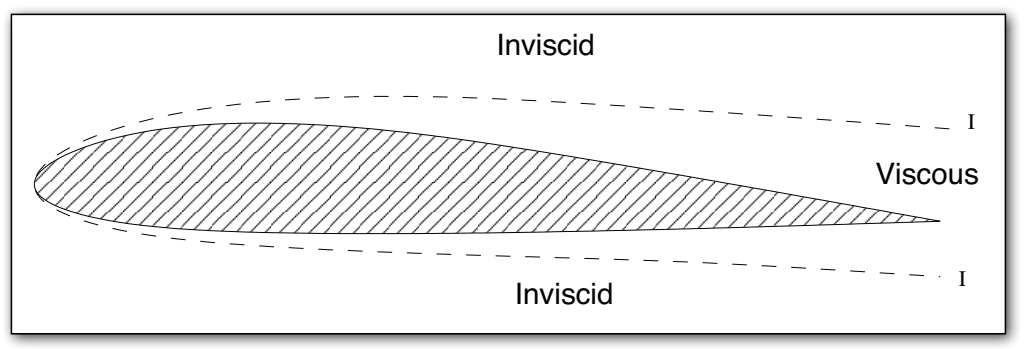

Wind farm aerodynamics

The description of the wind field in and around wind farms is especially relevant for large offshore wind farms, where the turbines will influence each other through their wakes. Planned offshore wind farms in the current UK Round 3 [9] have sizes from 400MW to 7000MW, which involve 80 to 1400 turbines with rated power of $5 \mathrm{MW}$. Wind turbines extract energy from the flow, leaving a wake behind where wind speed is reduced and turbulence is increased. The result is that a turbine operating in the wake of an upwind turbine will generate less power and will experience larger mechanical loads with respect to a turbine in ambient (undisturbed) wind conditions.

For the construction of large offshore wind farms, insight in wake effects is very important. The current tools available for the industry are so-called engineering tools, which are fast but lack accuracy. The Dutch research institutes ECN and CWI (Centre for Mathematics and Informat-

ics) take a more fundamental approach by developing CFD (computational fluid dynamics) methods and applying them to this problem, see review [10]. Researchers at ECN developed a tool (FarmFlow) that is a compromise between the fast but less accurate engineering tools and the more accurate but computationally expensive CFD tools.

Instead of solving the full 3D Navier-Stokes equations, both the diffusion and the pressure gradient in stream wise direction are neglected. This assumption effectively parabolizes the equations, allowing for a fast solution procedure by downstream marching. Furthermore, in order to include turbulence effects, the equations are averaged in time (Reynolds Averaged Navier-Stokes equations, RANS). The resulting FarmFlow software tool is an accurate and validated tool to calculate power losses in offshore wind farms. In addition, it calculates the added turbulence, which makes the tool unique, see Figure 5.

In order to get more insight in the physics of wind-turbine wakes and improve existing engineering models, improvements have to be made to the steady, parabolized approach. For example, to understand the fatigue loading on wind turbine blades, one has to capture the unsteady flow features in the wake of turbines, such as the tip vortices that originate from the blade tips. For this purpose, a full 3D unsteady incompressible Navier-Stokes solver is being constructed. This requires three main ingredients: - a numerical approximation (discretization) of the $\mathrm{Na}$ vier-Stokes equations in both space and time,

a turbulence model, and

- a representation of the turbine blades.

For the spatial and temporal discretisation of the incompressible Navier-Stokes equations we are looking at methods that inherit important properties of the continuous equations in a discrete sense, so-called 'mimetic', 'symmetry-preserving' or 'energy-conserving' methods [11]. Conserving energy in a discrete sense has three distinct advantages. Firstly, it leads to methods that are free of artificial (numerical) diffusion, which is of utmost importance when correctly simulating unsteady turbulent flows. Secondly, it leads to methods that are stable for any mesh at any time step, implying that the mesh and time step can be selected based on accuracy requirements rather than on stability. Thirdly, energy-conserving methods lead to well-behaved discretisation errors, even on coarse meshes and large time steps, because the discrete operators are well-posed. This is important, because simulations of turbulence are computationally very expensive and mesh sizes are kept as large as possible in practice.

To date, most work on energy-conserving methods has focused primarily on the spatial discretisation. An example of such a spatially energy-conserving method, which we employ in our work, is the staggered-grid method dating back to the work of Harlow and Welch [12]. We use this method and a higher-order extension of it on Cartesian (non-uniform) grids, for which it is particularly suitable. Recently, we have extended the notion of energy conservation to include time by employing a class of implicit Runge-Kutta methods [13a]. These methods allow stable and accurate time integration with large time 
